\title{
Memory effects in a nanoparticle system: Low-field magnetization and ac susceptibility measurements
}

\author{
R. K. Zheng, ${ }^{1}$ Hongwei Gu, ${ }^{2}$ Bing $\mathrm{Xu},{ }^{2}$ and X. X. Zhang ${ }^{1, *}$ \\ ${ }^{1}$ Department of Physics and Institute of Nano Science and Technology, The Hong Kong University of Science and Technology, \\ Clear Water Bay, Kowloon, Hong Kong, China \\ ${ }^{2}$ Department of Chemistry, The Hong Kong University of Science and Technology, Clear Water Bay, Kowloon, Hong Kong, China
}

(Received 28 August 2004; revised manuscript received 12 April 2005; published 8 July 2005)

\begin{abstract}
A dilute magnetic liquid with Co nanoparticles with an average diameter of $5 \mathrm{~nm}$ in hexane has been studied systematically following the experimental approach proposed recently for observing memory effects in magnetic nanoparticles. All phenomena reported previously have been observed, which were earlier ascribed to the memory effect. However, the standard experiments for observing memory effects (low frequency as susceptibility and low field de magnetization measurements) do not show the memory effect. To understand those observations, very detailed physical pictures, based on the relaxation of the individual particle, are proposed here.
\end{abstract}

DOI: $10.1103 /$ PhysRevB.72.014416

PACS number(s): 75.75.+a, 75.50.Lk, 75.50.Tt

\section{INTRODUCTION}

Because of the single domain nature of the magnetic nanoparticles (NPs), they are usually modeled as giant spin objects with significant anisotropy. The dynamics of isolated magnetic NPs have been successfully described by the thermally activated relaxation or superparamagnetism. Memory effect, a typical characteristic of spin-glass systems, ${ }^{1}$ has been observed and intensively studied in nanoparticle systems. ${ }^{2-6}$ Different experimental approaches have been employed to observe the memory effect in spin-glass system and particle systems. ${ }^{2-10}$ The most acceptable experimental approaches for observing the memory effect include low frequency ac susceptibility ${ }^{2-8}\left(\chi=\chi^{\prime}+\chi^{\prime \prime}\right)$ and low field dc magnetization measurements. ${ }^{5,6,9,10}$ The experimental details for each approach are briefly described as the following.

The low-frequency ac susceptibility approach includes four steps, (a) as the sample is cooling down from a temperature $\left(T_{H}\right)$ much higher than the freezing temperature of a spin-glass system at a constant rate, record the ac susceptibility; (b) stop at a temperature $\left(T_{1}\right)$ below $T_{F}$, wait for a period of time $t_{1}$ (a number of hours), and simultaneously measure $\chi$; (c) resume the cooling and record $\chi$ down to a base temperature, $T_{\text {base }}$; (d) heat the sample continuously from $T_{\text {base }}$ to the high temperature and record $\chi$.

The low-field dc magnetization approach includes three steps, (a) cool the sample in a zero-field with a constant rate from $T_{H}$; (b) stop at a temperature, $T_{0}$, below $T_{F}$ for $t_{1}$ then resume cooling down to $T_{\text {base }}$; (c) apply a small field at $T_{\text {base }}$ and measure $M$ with increasing temperature up to $T_{H}$. The difference between the resulting magnetization curve and a normal ZFC curve ${ }^{11}$ (without a stop at $T_{0}$ ) around $T_{0}$ can be ascribed to the memory effect.

The above methods have been regarded as the standard experimental approaches to observe the memory effect in spin-glass as well as in interacting nanoparticle systems. ${ }^{5-9}$

Very recently, Sun et al. performed a series of measurements on an interacting particle system and observed striking memory effects in the dc magnetization and magnetic relaxation. ${ }^{12}$ It has been suggested that the striking memory effects go beyond those previously observed in standard experiments and should be associated with a hierarchical model rather than a droplet model. The major difference between the standard experimental approaches and those used by Sun et al. is that during the sample cooling and waiting period no field is applied in the standard methods, but a nonzero field is applied in the sample cooling and then changed during the waiting in the Sun et al. experiments. It is well known that a field change in a particle system below its blocking temperature will cause a sharp change in the magnetization and a long-time, logarithmic relaxation, thereafter Ref. 11, which may suggest that the striking phenomena observed in Ref. 12 could be caused by the field change during waiting. ${ }^{13,14}$ To fully understand the mechanisms of the experimental approach, we replicated Sun et al. experiments on a dilute magnetic fluid with Co particles with an average diameter of 5 $\mathrm{nm}$ and observed the same phenomena. ${ }^{14}$

Since the particles are very weakly interacting or nearly noninteracting, their dynamics should be able to be described roughly by the pure thermal relaxation of individual particles rather than the collective dynamics (the memory effect). To confirm this, the standard approaches (low-frequency ac susceptibility and low-field dc magnetization measurements) have been employed on the same sample before and after the experiments. The results indicate that no memory effect is observed in the nearly noninteracting particle system when using the standard experimental methods. Therefore, the memory effect is not a unique interpretation to the phenomena observed by Sun et al. ${ }^{15}$ In addition, we conducted a simple numerical calculation by using the size distribution extracted from the experimental results, in which only the simple relaxation of the individual particles is considered. The calculated data show the same phenomena as observed experimentally. Although we briefly presented core physics as a comment, ${ }^{14}$ the full physical picture of the different experimental results has not been presented. In this paper, we give a clear picture of each experimental observation as reported in Ref. 12. 


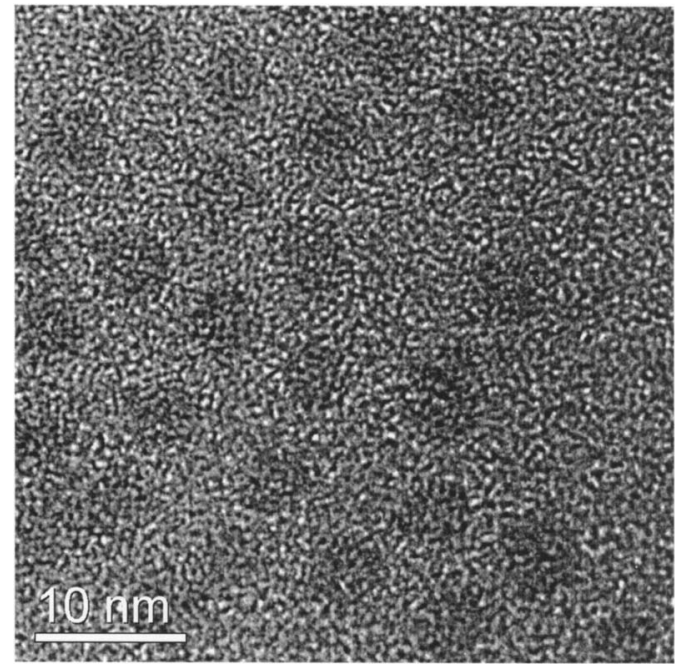

FIG. 1. The TEM image of the Co particles with an average size of $5 \mathrm{~nm}$ in diameter.

\section{EXPERIMENTS}

The sample used in this study is composed of Co nanoparticles synthesized by the chemical method. ${ }^{16}$ In an argonfilled glove box, $0.6 \mathrm{~g}$ of $\mathrm{Co}_{2}(\mathrm{CO})_{8}$ (in $3 \mathrm{~mL}$ of $o$-dichlorobenzene) was injected in a refluxing bath of $12 \mathrm{~mL}$ of $o$-dichlorobenzene (b.p., $182^{\circ} \mathrm{C}$ ) in the presence of 0.1 $\mathrm{mL}$ of oleic acid and $0.2 \mathrm{~g}$ of trioctylphosphine oxide (TOPO). After continuing the reflux for $30 \mathrm{~min}$, the heat source was then removed and the reaction mixture was allowed to cool to room temperature. The product was precipitated by adding ethanol $(\sim 10 \mathrm{~mL})$ and separated by centrifugation. A magnetic fluid was acquired after redispersal the precipitate in hexane $(\sim 10 \mathrm{~mL})$. The structure and the morphology of the particles were examined using the transmission electromicroscopy (TEM). The magnetic measurements were carried out on a Quantum Design superconducting quantum interference device (SQUID) magnetometer (MPMS-5s) with an ac susceptibility option in the temperature range from 10 to $150 \mathrm{~K}$.

\section{RESULTS AND DISCUSSION}

Shown in Fig. 1 is a typical TEM image of the Co particles. The TEM image shows that the particles have a nearly spherical shape and an average diameter of $5 \mathrm{~nm}$. To characterize the sample magnetically, we first measured the zerofield-cooled (ZFC) and field-cooled (FC) magnetization in a 100 Oe magnetic field on the diluted magnetic fluid with a temperature sweeping rate of $2 \mathrm{~K} / \mathrm{min}$. Since the liquid carrier is hexane, which has a freezing point of about $165 \mathrm{~K}$, we cooled the sample from $300 \mathrm{~K}$ to the base temperature $10 \mathrm{~K}$ in a zero field and then all the measurements were limited below $150 \mathrm{~K}$ to guarantee that the sample was solid and to keep the particles fixed physically. Shown in Fig. 2 are the $\mathrm{ZFC}$ and FC magnetization curves. The ZFC curve peaks at $35 \mathrm{~K}$, which corresponds to the average blocking temperature $\left(\left\langle T_{B}\right\rangle\right)$ of the system at $100 \mathrm{Oe}$ and the FC curve monotonically decreases with increasing temperature. To examine

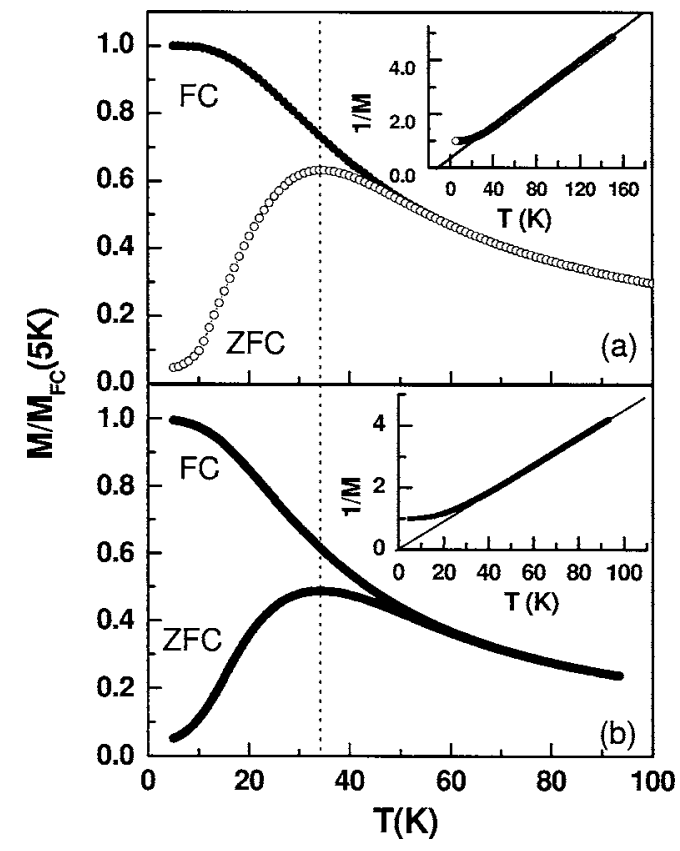

FIG. 2. The measured and simulated zero-field-cooled and fieldcooled magnetization curves in 50 Oe field. The magnetization is normalized to $M_{\mathrm{FC}}(5 \mathrm{~K})$. The insets show the reciprocal of the normalized $M_{\mathrm{FC}}$ as a function of temperature.

the interaction between the particles, we plotted $1 / M$ versus $T$ for the FC curve, as shown in the inset of Fig. 2.

The perfect linearity of the curve for $T \geqslant 35 \mathrm{~K}$ indicates that the dynamics of the particles above $\left\langle T_{B}\right\rangle$ can be described by superparamagnetism, a behavior of the individual particles. In addition, the FC curve represents the thermal equilibrium states of the sample. Therefore, the curve is described by the Curie-Weiss law, $\chi=\frac{M}{H}=\frac{C}{(T-\theta)}$. By fitting the data to the Curie-Weiss law, we find that $\theta=-10 \mathrm{~K}$. The small negative value of $\theta$ suggests that a weak antiferromagnetic-type of dipole-dipole interaction exists in the system. The strength of the interaction obtained from the Curie-Weiss law can be considered as a mean interaction in the system, because a disordered system should have a distribution of interaction. This interaction can also be obtained by using Vogel-Fulcher law to analyze ac susceptibility data measured with different frequencies, which usually gives a very similar value as Curie-Weiss law. ${ }^{17}$ Since the sample is very dilute, the interaction might suggest that it is not completely homogenous and contains small agglomerates. ${ }^{17,18}$ Another important feature is that the FC curve departs from the $\mathrm{ZFC}$ curve at about $50 \mathrm{~K}$, which is much larger than $\left\langle T_{B}\right\rangle=35 \mathrm{~K}$. Actually, this departure temperature of the ZFC and FC curves should correspond to the maximum blocking temperature in the system. This implies the existence of an energy barrier distribution in the sample due to the size variation.

In addition to the dipole-dipole interaction, CasimirPolder-type Van de Waals interaction has recently been observed in the magnetic nanoparticle systems. ${ }^{19}$ However, we believe that the Casimir-Polder-type Van de Waals interaction might not exist in our sample or be too week to be 


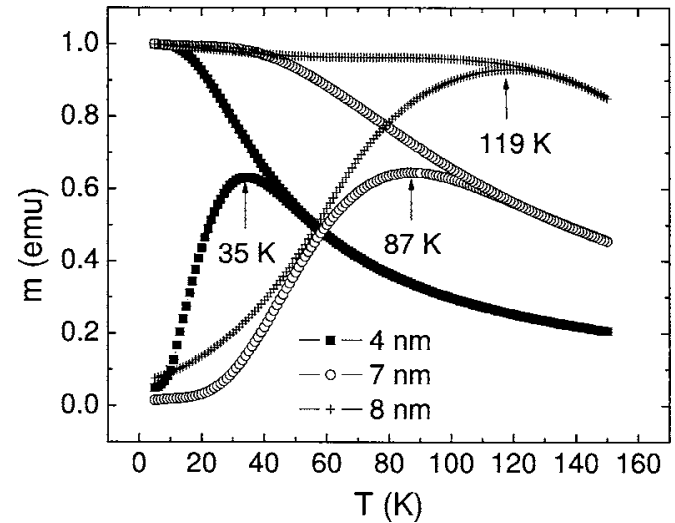

FIG. 3. ZFC-FC curves of Co particles of different sizes. The blocking temperature increases with increasing the particle size.

detected, because we did find that the blocking temperature increases with the particles size (Fig. 3), and because we did not find a halt in the magnetic relaxation curves measured in temperature range of $1.8 \mathrm{~K}$ up to the blocking temperature (Fig. 4). ${ }^{19}$ Therefore, the sample might be considered as nearly noninteracting.

The interaction in our sample is very samll in comparison with the anisotropy energy $U$, which can be estimated by using $U / k_{B}=25 T_{B}$, where $T_{B}$ is the blocking temperature. In this sample, $T_{B}$ is about $35 \mathrm{~K}$, the anisotropy energy will be about several hundred $\mathrm{K}$, being much larger than the interaction. Therefore the behavior of the particle can be considered as nearly noninteraction. Another evidence is that the interaction is so small that the perfect linearity of $1 / M_{\mathrm{FC}}$ for $T \geqslant 35 \mathrm{~K}$ (the blocking temperature) indicates that the dynamics of the particles above $\left\langle T_{B}\right\rangle$ can be described by superparamagnetism, a behavior of the individual particles.

The most striking experimental observation reported by Sun et al. is the steplike behavior of the $\mathrm{M}(\mathrm{T})$ curves (Fig. 2 of Ref. 12). These curves were obtained from the following procedure. The sample was cooled in 50 Oe field with a constant cooling rate of $2 \mathrm{~K} / \mathrm{min}$ from $T_{H}$ to $T_{\text {base }}$. After reaching $T_{\text {base }}$, the sample was heated continuously at the same temperature sweep rate $(2 \mathrm{~K} / \mathrm{min})$ to $T_{H}$. The obtained $M(T)$ curves are the normal FC curve, which can be considered as a good approximation of the thermal equilibrium

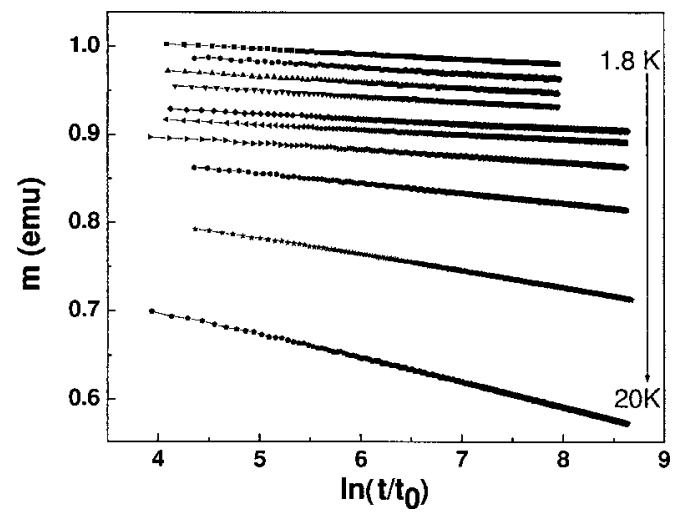

FIG. 4. Relaxation data obtained on the Co particles of $5 \mathrm{~nm}$ in diameter. No halt in relaxation is observed.
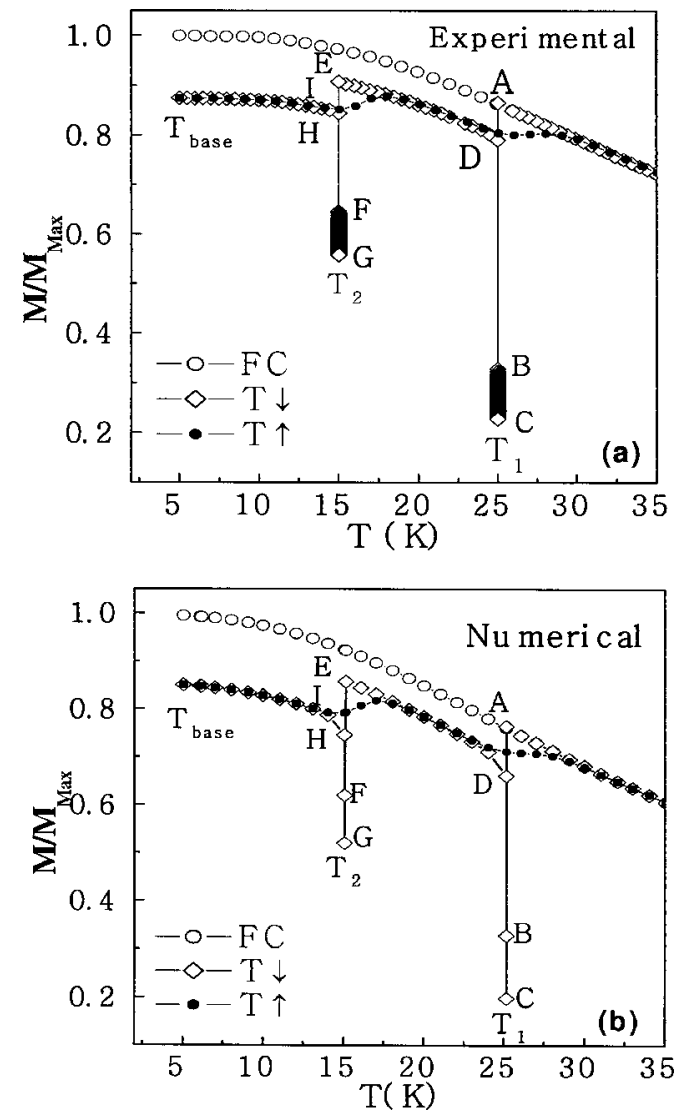

FIG. 5. The temperature dependent magnetization curves [normalized to $M_{\mathrm{FC}}(5 \mathrm{~K})$ ] obtained in 50 Oe field. (a) The experimental data; (b) the numerical data.

states. Then, the sample was cooled again at the same rate, but the cooling was stopped temporarily three times below $\left\langle T_{B}\right\rangle$ with a pause of $t_{w}=4 \mathrm{~h}$ at each stop. During each temporary stop, the applied field was also turned off to let the sample relax. After the pauses, the applied field was reapplied and the cooling was resumed. The $M(T)$ curve obtained with the temporary pause shows the steplike behavior. Once the temperature reached $T_{\text {base, }}$ the sample was warmed continuously at the same rate to $T_{H}$. The $M(T)$ curve obtained upon warming also shows the steplike behavior around each stopping temperature. Sun et al. suggested that the steplike behavior observed during reheating is due to the memory effect, and that the memory effect indicates that there is a hierarchical organization of metastable states resulting from significant interparticle interactions. It is well known that the hierarchical picture proposed for the spin-glass system requires a large number of degree of freedom to be coupled. The memory effect, thus, cannot be produced by a simple thermal relaxation. However, we believe the steplike $M(T)$ curves may not be just due to the memory effect, because the same behavior can be observed in a noninteracting particle system if the same experiments are performed.

Shown in Fig. 5(a) is the $M(T)$ curve obtained on our Co particles following the above procedure, with $t_{w}=3 \mathrm{~h}$, a temperature rate of $1 \mathrm{~K} / \mathrm{min}, T_{H}=150 \mathrm{~K}, T_{\text {base }}=5 \mathrm{~K}$ and a 100 Oe applied field. As expected the steplike behavior of $M(T)$ is clearly seen and all the features of the curve are obviously 
the same as those reported in Ref. 12. Most importantly, we demonstrate that the steplike behavior has also been observed in a noninteracting particle system when using the experimental approach used by Sun et al. We show later that this system does not indicate the memory effect if we use the standard experimental approaches for observing the memory effect.

Actually, the steplike behavior shown in Fig. 5(a) can easily be accounted for from the simple thermal relaxation of the individual particles. For simplicity, we consider a particle system as single domain particles with a uniaxial anisotropy and a size distribution and assume that the easy axes of the anisotropic particles are aligned in the same direction. For a field much smaller than the anisotropy field, $H_{\text {an }}$, applied along the easy axis, the dynamics of each particle is governed by the energy barrier, $U(H)=K V\left(1-H / H_{\text {an }}\right)^{2} \approx K V$ (for example, $H=100 \mathrm{Oe}$ and $H_{\mathrm{an}}=10000 \mathrm{Oe}$ ) and the temperature, $T$, because the reversal rate of the magnetic moment follows Arrhenius law,

$$
\Gamma=\Gamma_{0} \exp \left[-U(H) / k_{B} T\right] .
$$

For each particle, therefore, there is a blocking temperature, $T_{B}$, at which the relaxation time, $1 / \Gamma$, is equal to the time of one measurement, $t_{m}$, i.e.,

$$
t_{m}=1 / \Gamma=\left(1 / \Gamma_{0}\right) \exp \left[U(H) / k_{B} T_{B}\right] .
$$

For a ferromagnetic particle, $\Gamma_{0}=10^{9}-10^{10} \mathrm{~s}^{-1}$ and $t_{m}=30 \mathrm{~s}$ (for a SQUID magnetometer), we have $U=K V \approx 25 k_{B} T_{B}$. Above $T_{B}$, the particles is superparamagnetic and reacts immediately to a field change, whereas when $T<T_{B}$, the reversal rate of the moment is determined by Eq. (1). For a distribution in $V$, there must be a similar distribution in $T_{B}$. The anisotropy constant of the particles has been calculated to be $1.8 \times 10^{6} \mathrm{emu} / \mathrm{cm}^{3}$ with the average diameter of $5 \mathrm{~nm}$ and $\left\langle T_{B}\right\rangle=35 \mathrm{~K}$, which is in agreement with the value reported previously. ${ }^{17,20}$

It is shown that the ZFC and FC curves can be numerically obtained based on the thermal relaxation using a given size distribution and the anisotropy constant. ${ }^{21}$ Shown in Fig. 2(b) are the numerically calculated ZFC and FC curves using the size distribution and the anisotropy constant extracted from the ZFC curve in Fig. 2(a).

It is clearly seen that a peak appears in ZFC curve. The peak temperature is the average blocking temperature, $\left\langle T_{B}\right\rangle$. The FC curve was obtained by cooling the sample from $T_{H}$, where all the particles are superparamagnetic (or in thermal equilibrium state). For a cooling rate of a few $\mathrm{K} / \mathrm{m}$, the $\mathrm{FC}$ curve $\left(M_{\mathrm{FC}}\right)$ should be a good approximation of the equilibrium state $\left(M_{\text {eq }}\right)$. In both the ZFC and FC curves, with increasing $T$, particles with $T_{B} \leqslant T$ become superparamagnetic and reach their equilibrium and their contribution to $M$ is given by Curie law, i.e., 1/T. The increase in ZFC for $T$ below $\left\langle T_{B}\right\rangle$ is due to the relaxation of the particles whose blocking temperatures are not much higher than $T$, because the relaxation rate [Eq. (1)] is extremely small for larger particles. As $T$ increases a peak must appear in $\mathrm{ZFC}$ at $\left\langle T_{B}\right\rangle$, due to the $1 / T$ dependence of $M$ when $T$ is higher than the average blocking temperature.
We also performed a numerical experiment following the procedures used by Sun et al. to obtain the results shown in Fig. 5(b), which are basically the same as the experimental results in Fig. 5(a). Our results demonstrate that the steplike $M(T)$ can also be observed with the nearly noninteracting particles both experimentally and numerically.

Now let us interpret the results using the thermal relaxation model. Since the numerical curves not only mimic the experimental results, but also give all the details of the curve at any temperature and time, we will follow the numerical results [Fig. 5(b)] to interpret the phenomena. It is evident that the cooling curve from $T_{H}$ to $T_{1}=25 \mathrm{~K}$ (point $\mathbf{A}$ ) in a magnetic field $(H=100 \mathrm{Oe})$, at which the cooling is temporarily stopped and the applied field is cut off as well, follows FC. After $H$ is cut off, $M$ drops sharply to point $\mathbf{B}$, by the amount of the contribution of particles with $T_{B} \leqslant T_{1}$, because the moment reversal for particles with $T_{B}>T_{1}$ occurs only through the activation process. During the waiting time $(3 \mathrm{~h})$, $M$ decreases logarithmically to point $\mathbf{C}$ through the relaxation of the particles with $T_{B}$ being not much larger than $T_{1}$. After field $H$ is turned on, $M$ increases immediately to point $\mathbf{D}$, but cannot recover its original value at point $\mathbf{A}$ in a short time, because the moments of the particles that flipped down during the pause can flip back only through the slow thermal activation. As $T$ decreases further, the relaxation rate for the reversed particles decreases exponentially and only small portion of the particles relaxes up at $T$ near $T_{1}$. Consequently, the overall behavior for the cooling curve (point $\mathbf{D}$ to point E) is very similar to the FC curve. Similarly, the behavior of the curves at $T_{2}$ (from point $\mathbf{E}$ to point $\mathbf{H}$ ) shows is the same as observed at $T_{1}$. The curve from point $\mathbf{H}$ to $T_{\text {base }}=5 \mathrm{~K}$ is due to the same mechanism as is the curve from point $\mathbf{D}$ to point $\mathbf{E}$.

Similarly, the warming curve from $T_{\text {base }}$ will be also very similar to the cooling curve at low $T$ due to the very slow relaxation rate for the larger particles with $T_{B}>T_{2}$. Only when $T$ is approaching $T_{2}$, does the relaxation rate become significant for the reversed particles at $T_{2}$, and $M$ begins to increase. The slight decrease in the warming curve from $T_{\text {base }}$ to point $\mathbf{I}$ is due to the same mechanism as in the FC curve. Since the significant relaxation of the reversed particles begins slightly below $T_{2}, M$ must be higher than the point $\mathbf{H}$, and increases smoothly as in the ZFC curve. Finally, $M$ recovers its value between $T_{2}$ and $T_{1}$. As the temperature increases and approaches to $T_{1}$, the relaxation rate becomes significant for the reversed particles at $T_{1}$, and $M$ begins to increase as it did around $T_{2}$ and finally it recovers the value corresponding to the FC curve. It is obvious that the steplike behavior obtained in the noninteracting particles can be interpreted in the context of the thermal activation of the individual particles, and the interaction between the particles is absolutely not essential to producing the steplike curves.

It might be argued that our experimental observations may also be due to the memory effect. To determine if this could be the case, we made the low-frequency ac susceptibility and low-field magnetization measurements using the standard approach ${ }^{2-10}$ before and after the experiments reported in Fig. 5. The results in Fig. 6 are the low-field dc measurements. ${ }^{2}$ It is clearly seen that all the curves are superimposed (coincident with each other), and that no 


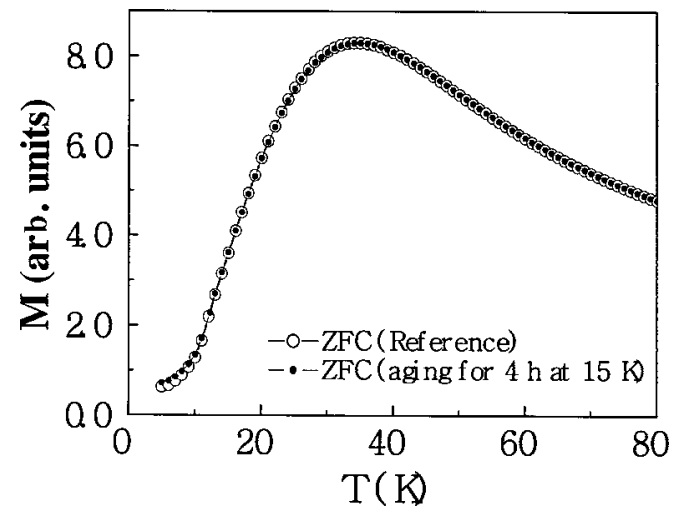

FIG. 6. Low field of ZFC magnetization curves obtained with the standard experimental approach for observing the memory effect.

memory effect is observed. Figure 7 gives the low-frequency ac susceptibility measurements following the standard procedure. ${ }^{2-8}$ The cooling during the $\chi(T)$ measurement is temporarily stopped at $15 \mathrm{~K}$ for $t_{w}=7 \mathrm{~h}$. Again, no memory effect is indicated by the curves. We believe that the steplike $M(T)$ curves shown in Fig. 5(a) should not be due to the memory effect. Moreover, the weak interaction (it is about $10 \mathrm{~K}$ in this study) among the particles is unlikely to cause a spin-glass phase.

Furthermore, almost identical results to those shown in Figs. 3-5 in Ref. 12 have been experimentally and numerically replicated with our noninteracting Co particle sample. Shown in Fig. 8(a) are the relaxation measurements from the Sun et al. ZFC method. The sample is first zero-field cooled from $100 \mathrm{~K}$ to $20 \mathrm{~K}$. When a field of $50 \mathrm{Oe}$ is applied, the magnetization immediately reaches a certain value (point $\mathbf{A}$ ) as determined by the equilibrium magnetization of the particles with $T_{B} \leqslant 20 \mathrm{~K}$. Then, a slow logarithmic relaxation begins as shown in the inset of Fig. 8(a). The logarithmic relaxation is due to the energy distribution resulting from the size distribution of the particles. ${ }^{22}$ The relaxation was recorded for $8000 \mathrm{~s}\left(t_{1}\right)$, point $\mathbf{A} \rightarrow$ point $\mathbf{B}$. After $t_{1}$, the sample is quenched to $5 \mathrm{~K}$ under the constant field and the relaxation was recorded for $8000 \mathrm{~s}\left(t_{2}\right)$, point $\mathbf{C} \rightarrow$ point $\mathbf{D}$. The sudden increase in magnetization (point $\mathbf{B}$ to point $\mathbf{C}$ ) is

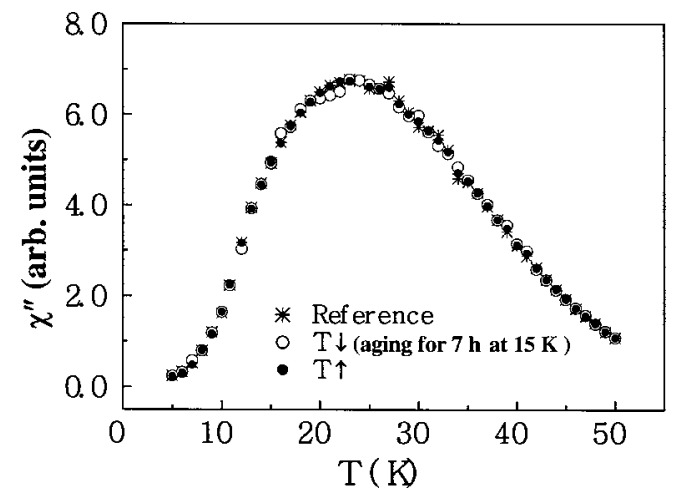

FIG. 7. Low frequency $(f=0.04 \mathrm{~Hz})$ susceptibility data obtained with the standard experimental approach for observing the memory effect.
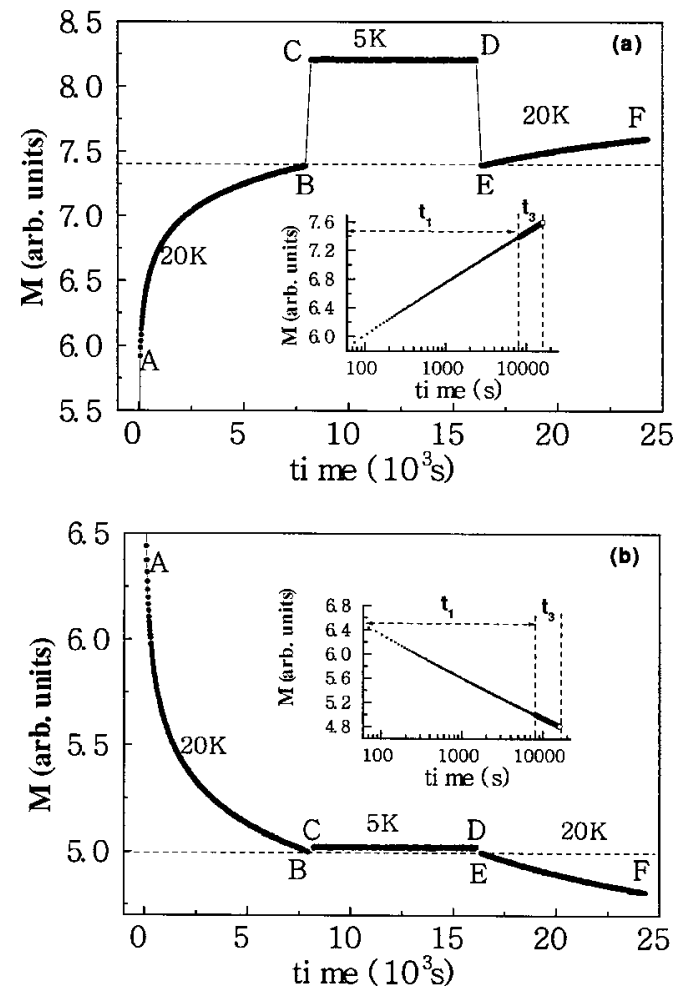

FIG. 8. Magnetic relaxation data obtained in a constant field of 50 Oe, but with a temporary cooling, for (a) the ZFC method and (b) the FC method. The inset shows the data during $t_{1}$ and $t_{3}$ in the logarithmic time, where the data in $t_{3}$ is plotted as a function of $t$ $-t_{2}$

due to the particles with $T_{B} \leqslant 20 \mathrm{~K}$ and those that flipped during $t_{1}$ reach their new equilibrium state at $5 \mathrm{~K}$ as in the FC case. Since only the particles with $T_{B}>20 \mathrm{~K}$ are not in the equilibrium state and relax extremely slowly at $5 \mathrm{~K}$, the curve recorded at $5 \mathrm{~K}$ is almost constant. Finally, the sample was heated to $20 \mathrm{~K}$ and then allowed to relax for another $8000 \mathrm{~s}\left(t_{3}\right)$, point $\mathbf{E} \rightarrow$ point $\mathbf{F}$. When the sample is heated back to $20 \mathrm{~K}$, the particles with $T_{B}<20 \mathrm{~K}$ and those relaxed during $t_{1}+t_{2}$ return to the equilibrium state at point $\mathbf{B}$ (or point $\mathbf{E}$ ), because they approximately follow the equilibrium curve, i.e., the $\mathrm{FC}(w)$ curve. Therefore it is evident that the relaxation in $t_{3}$ will be the continuation of the curve in $t_{1}$, the inset of Fig. 8(a). It is also obvious that the phenomena can be interpreted by the thermal activation events of the individual particles.

The results shown in Fig. 8(b) were obtained through the FC method, which can be similarly interpreted by the pure thermal activation of the individual particles. After the sample was first cooled in a 50 Oe field from $100 \mathrm{~K}$ to $20 \mathrm{~K}$, the field was cut off and the magnetization was recorded for $t_{1}=8000 \mathrm{~s}$, point $\mathbf{A} \rightarrow$ point $\mathbf{B}$. At $20 \mathrm{~K}$, after the field was cut off, particles with $T_{B} \leqslant 20 \mathrm{~K}$ do not contribute to the measured magnetization, because their equilibrium state has zero magnetization. This explains the sharp drop in $M$ after the field was cut off. The logarithmic relaxation results from the particles with $T_{B}>20 \mathrm{~K}$. After $t_{1}$, the sample was quenched to $5 \mathrm{~K}$ in the zero-field and the magnetization was recorded for $t_{2}=8000 \mathrm{~s}$ at $5 \mathrm{~K}$, point $\mathbf{C} \rightarrow$ point $\mathbf{D}$. As the 


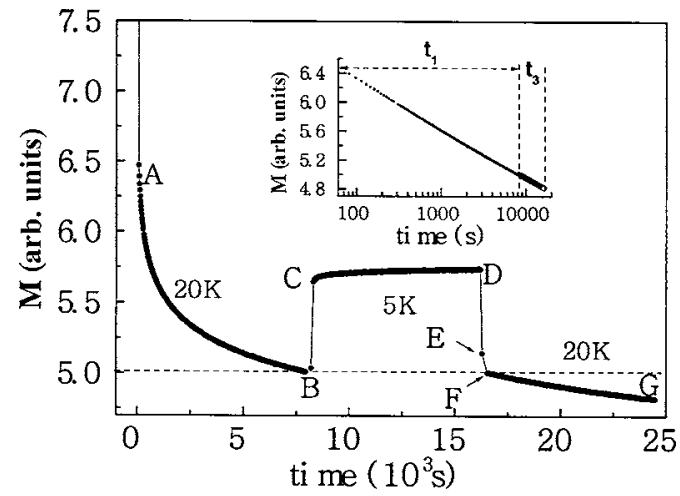

FIG. 9. Magnetic relaxation data obtained with a field change during the temporary cooling for the FC method. The inset shows the data during $t_{1}$ and $t_{3}$ in the logarithmic time, while the data in $t_{3}$ is plotted as a function of $t-t_{2}$.

sample is quenched to $5 \mathrm{~K}$, the remnant magnetization increases very slightly due to the reduced thermal agitation of the magnetization. Again, since the relaxation rate, determined by Eq. (1) for each particle with $T_{B}>20 \mathrm{~K}$, is extremely small, the curve measured during $t_{2}$ is almost flat. Finally, the sample was heated back to $20 \mathrm{~K}$ and the magnetization was measured for another time $t_{3}=8000 \mathrm{~s}$, point $\mathbf{E}$ $\rightarrow$ point $\mathbf{F}$. When the particles are heated back to $20 \mathrm{~K}$, the magnetization returns to the value before the quenching, because almost no flipping occurs at low $T$. Obviously the curve in $t_{3}$ must follow the curve in $t_{1}$, as shown in the inset of Fig. 8(b).

We also performed the experiments using the methods employed by Sun et al. to obtain the results shown in Fig. 4 of Ref. 12. We observed basically the same phenomena, as shown in Fig. 9. For the FC method, after the sample being cooled from $100 \mathrm{~K}$ in a 50 Oe field to $20 \mathrm{~K}$, the field is cut off, and the magnetization is measured for $t_{1}$ $=8000 \mathrm{~s}$, point $\mathbf{A} \rightarrow$ point $\mathbf{B}$. The measured magnetization is the same as that for time $t_{1}$ in Fig. 8(b). The difference here is that after the sample is quenched to $5 \mathrm{~K}$ in the zero field, a 50 Oe field is turned on. The sharp jump in $M$, point $\mathbf{B}$ $\rightarrow$ point $\mathbf{C}$, results from the increase in magnetization of the particles with $T_{B} \leqslant 5 \mathrm{~K}$. During $t_{2}$, point $\mathbf{C} \rightarrow$ point $\mathbf{D}$, the magnetization increases due to the relaxation of the particles whose $T_{B}$ is not much greater than $5 \mathrm{~K}$. After $t_{2}$, the field was turned off, and the magnetization jumped down from point $\mathbf{D}$ to point $\mathbf{E}$. A closer examination reveals that the change from point $\mathbf{B}$ to point $\mathbf{C}$ is almost equal to the change from point $\mathbf{D}$ to point $\mathbf{E}$, because both are due to the fact that the particles with $T_{B} \leqslant 5 \mathrm{~K}$ reach their thermal equilibrium immediately following the field change. When the temperature is raised to $20 \mathrm{~K}$, the particles that relax during $t_{2}$ flip back during the temperature increases from $5 \mathrm{~K}$ to $20 \mathrm{~K}$, point $\mathbf{E}$ to point $\mathbf{F}$. After the temperature loop, the system returned to its state before the quenching. Again the curve in $t_{3}$, Point $\mathbf{F}$ to point $\mathbf{G}$, must be a continuation of the curve in $t_{1}$, as shown in the inset of Fig. 9.

Similarly, the results shown in Fig. 4(a) and Fig. 5 of Ref. 12 , can be easily understood in the context of the thermal relaxation of the individual particles.

\section{CONCLUSION}

In summary, we have performed experiments on nearly noninteracting Co particles by using the same experimental scheme described in Ref. 12 and reproduced all the phenomena presented there. We have also described a very detailed picture and explained all the phenomena based on the thermal relaxation model of individual particles. Therefore, the observations presented in Ref. 12 are not sufficient proof for memory effect, at least, in particle systems.

\section{ACKNOWLEDGMENT}

The work described in this paper was fully supported by grants from the Research Grants Council of the Hong Kong Special Administration Region, China.
*Electronic address: phxxz@ust.hk

${ }^{1}$ K. Binder and A. P. Young, Rev. Mod. Phys. 58, 801 (1986).

${ }^{2}$ H. Mamiya, I. Nakatani, and T. Furubayashi, Phys. Rev. Lett. 82, 4332 (1999).

${ }^{3}$ P. Jonsson, M. F. Hansen, and P. Nordblad, Phys. Rev. B 61, 1261 (2000).

${ }^{4}$ P. Jonsson, M. F. Hansen, P. Svedlindh, and P. Nordblad, Physica B 284, 1754 (2000).

${ }^{5}$ S. Sahoo, O. Petracic, W. Kleemann, P. Nordblad, S. Cardoso, and P. P. Freitas, Phys. Rev. B 67, 214422 (2003).

${ }^{6}$ O. Cador, F. Grasset, H. Haneda, and J. Etourneau, J. Magn. Magn. Mater. 268, 232 (2004).

${ }^{7}$ K. Jonason, E. Vincent, J. Hammann, J. P. Bouchaud, and P. Nordblad, Phys. Rev. Lett. 81, 3243 (1998).

${ }^{8}$ V. Dupuis, E. Vincent, J. P. Bouchaud, J. Hammann, A. Ito, and H. A. Katori, Phys. Rev. B 64, 174204 (2001).

${ }^{9}$ R. Mathieu, P. Jonsson, D. N. H. Nam, and P. Nordblad, Phys.
Rev. B 63, 092401 (2001).

${ }^{10}$ R. Mathieu, P. E. Jönsson, P. Nordblad, H. Aruga Katori, and A. Ito, Phys. Rev. B 65, 012411 (2001).

${ }^{11}$ X. X. Zhang, J. M. Hernandez, J. Tejada, and R. F. Ziolo, Phys. Rev. B 54, 4101 (1996).

${ }^{12}$ Y. Sun, M. B. Salamon, K. Garnier, and R. S. Averback, Phys. Rev. Lett. 91, 167206 (2003).

${ }^{13}$ M. Sasaki, P. E. Jonsson, H. Takayama, and P. Nordblad Phys. Rev. Lett. 93, 139701 (2004).

${ }^{14}$ R. K. Zheng, Hongwei Gu, and X. X. Zhang, Phys. Rev. Lett. 93, 139702 (2004).

${ }^{15}$ Y. Sun, M. B. Salamon, K. Garnier, and R. S. Averback, Phys. Rev. Lett. 93, 139703 (2004).

${ }^{16}$ S. Sun and C. B. Murray, J. Appl. Phys. 85, 4325 (1999).

${ }^{17}$ X. X. Zhang, G. H. Wen, G. Xiao, and S. H. Sun, J. Magn. Magn. Mater. 261, 21 (2003).

${ }^{18}$ C. Djurberg, P. Svedlindh, P. Nordblad, M. F. Hansen, F. Bødker, 
and S. Mørup, Phys. Rev. Lett. 79, 5154 (1997).

${ }^{19}$ R. V. Chamberlin, J. Hemberger, A. Loidl, K. D. Humfeld, D. Farrell, S. Yamamuro, Y. Ijiri, and S. A. Majetich, Phys. Rev. B 66, 172403 (2002).

${ }^{20}$ S. I. Woods, J. R. Kirtley, Shouheng Sun, and R. H. Koch, Phys. Rev. Lett. 87, 137205 (2001).
${ }^{21}$ J. C. Denardin, A. L. Brandl, M. Knobel, P. Panissod, A. B. Pakhomov, H. Liu, and X. X. Zhang, Phys. Rev. B 65, 064422 (2002).

${ }^{22}$ J. Tejada, X. X. Zhang, and E. M. Chudnovsky Phys. Rev. B 47, 14977 (1993). 